FM 16:

Stellar Behemoths - Red Supergiants across the local Universe 


\title{
Type II supernova diversity
}

\author{
Joseph P. Anderson \\ European Southern Observatory, Alonso de Crdova 3107, Casilla 19001, Santiago, Chile \\ email: janderso@eso.org
}

\begin{abstract}
It is now firmly established that at a significant fraction of hydrogen-rich type II supernovae (SNe II) arise from red supergiant progenitors. However, a large diversity of SN properties exist, and it is presently unclear how this can be understood in terms of progenitor differences and pre-SN stellar evolution. In this contribution, I present the diversity of SN II $V$-band light-curves for a large sample of SNe II, and compare these to photometry of SNe II which have progenitor mass constraints from pre-explosion imaging.
\end{abstract}

Keywords. (stars:) supernovae: general

Type II supernovae (SNe II) are the explosions of massive (>8-10 $\left.\mathrm{M}_{\odot}\right)$ stars which have retained a significant fraction of their hydrogen envelopes. Historically, these events were separated into 'Plateau' IIPs which show almost constant luminosity for $\sim 100$ days, and 'Linear' IIL which decline much faster in a linear manner (Barbon, Ciatti \& Rosino 1979). However, recent work has questioned this separation and argued for a continuum of events with very few (if any) SNe showing the historically defined morphology of a SN IIL (Anderson et al. 2014; Sanders et al. 2014). The direct identification of red supergiant (RSG) progenitors on pre-explosion images (e.g. Smartt 2015) is concrete evidence that SNe II arise from the explosions of RSG stars. However, it is unclear how the large diversity of SN II light-curves and spectra is linked to progenitor properties such as mass, metallicity, extent of the pre-explosion hydrogen envelope, or the role of binary interactions. Analysing the diversity within large samples of events, and searching for correlations between parameters to try to understand the whole ensemble of explosions through consistent analyses can aid in our understanding. This was recently achieved in Anderson et al. (2014, A14), and this contribution I briefly summarise some of the important results from that work, while comparing those light-curves to the same photometric measurements for SNe II with progenitor mass constraints.

In Fig. 1a I present a schematic outlining the $V$-band light-curve parameters chosen in A14 to characterise the photometric diversity of SNe II. These include a range of absolute magnitudes at different phases; a range of decline rates; and various time epochs and time durations. Some of these parameters were previously well documented, however others were not (see A14 for a detailed discussion). In particular, the early decline rates ( $s_{1}$ in our nomenclature) have received little discussion in the literature. In A14 we also highlight differences between the optically thick phase duration (OPTd, historically named the plateau duration): the time between explosion and the end of the plateau, and the plateau duration $(\mathrm{Pd})$ which we now define as the time duration for which a SN is actually showing a 'plateau', i.e. from the end of the initial $s_{1}$ decline to the end of $s_{2}$ when the SN starts transitioning to the later radioactively powered phase.

All photometric parameters defined in Fig. 1a were measured (where possible) for the full sample of $>100$ SNe II in A14. The same parameters are also measured for all those SNe II with progenitor mass constraints. In Fig. 1b the absolute $V$-band magnitude lightcurves for both the A14 and direct detection samples are presented. This figure clearly shows the large diversity seen in SN II light-curves. Diversity is observed in terms of 

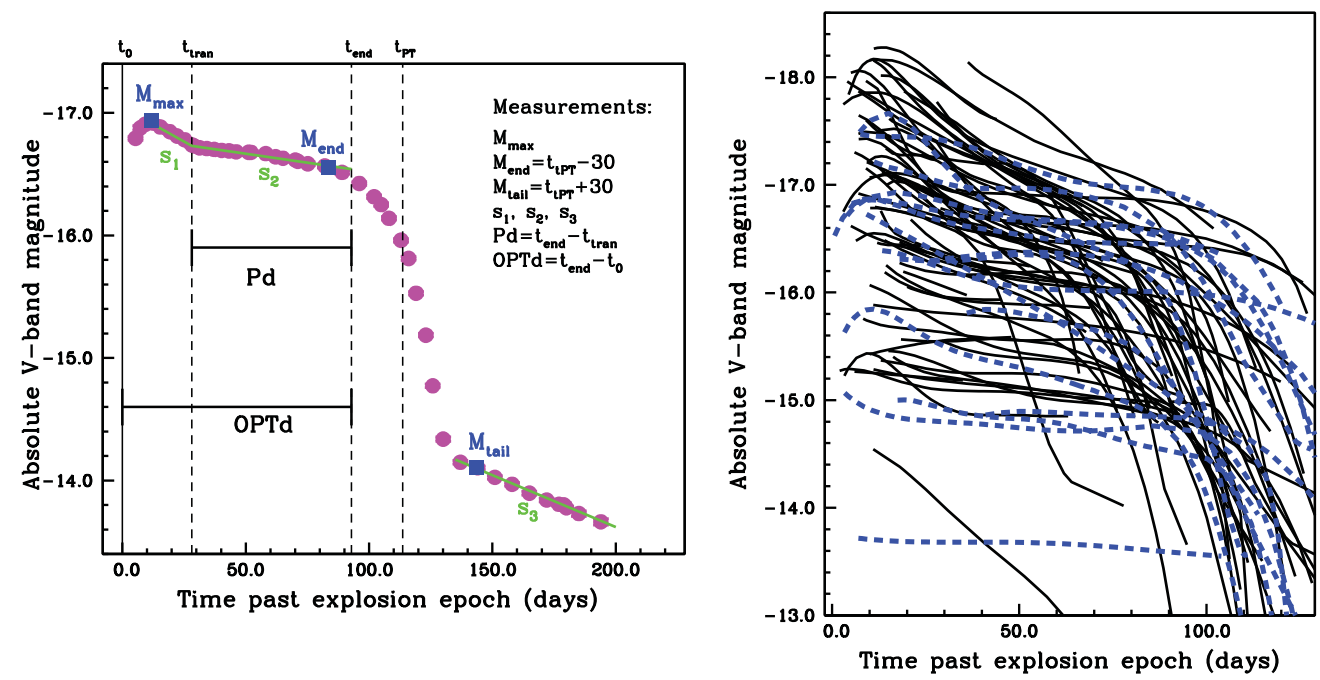

Figure 1. Left, $\boldsymbol{a}$ ): SN II light-curve schematic outlining the $V$-band photometric parameters defined and measured for all SNe II in this work. Right, $\boldsymbol{b}$ ): absolute $V$-band light-curves of $\mathrm{SNe}$ II. Those in solid lines are from A14, while those in dashed lines are SNe II with direct progenitor detection constraints in the literature.
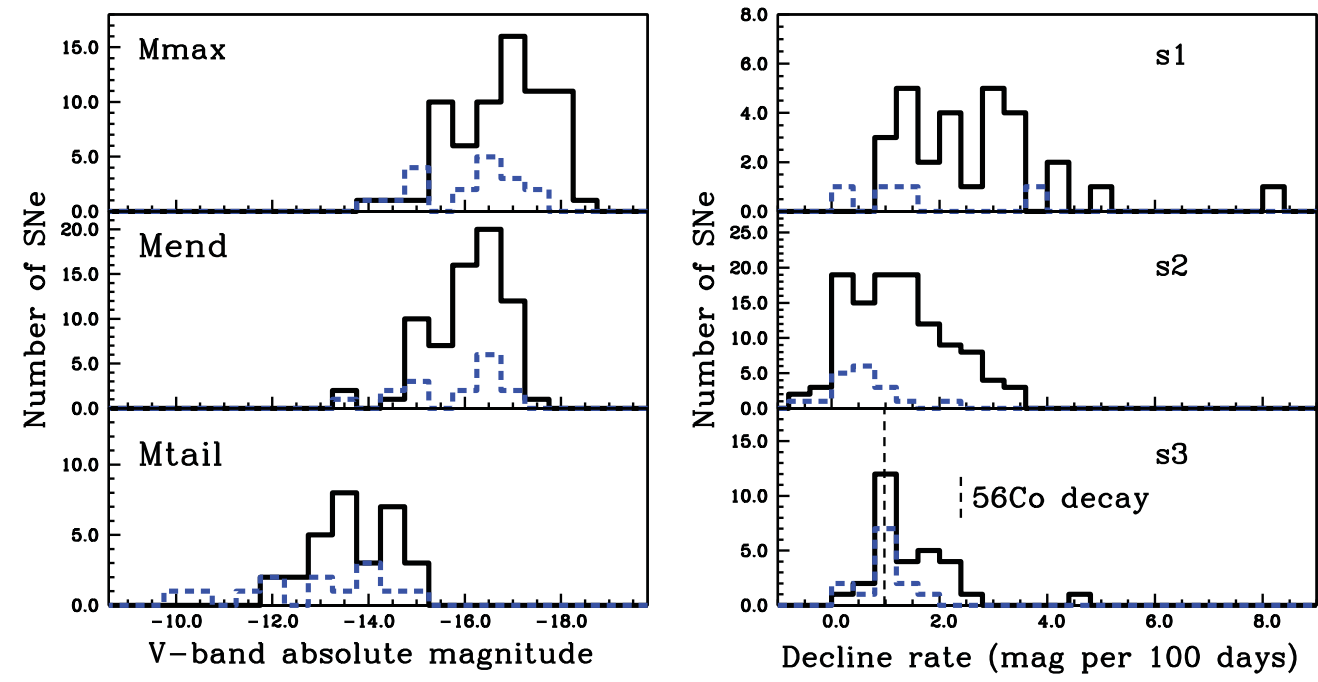

Figure 2. Left, $\boldsymbol{a}$ ): Histograms of the three absolute magnitudes of SNe II. Right, $\boldsymbol{b}$ ): Histograms of the three decline rates of SNe II. Those in solid lines are from A14, while the dashed lines are $\mathrm{SNe}$ with progenitor detection constraints.

brightness, decline rates, and optically thick phase durations. The direct detection sample also spans a wide range of light-curve morphologies. This is interesting, especially in the context of their distribution of progenitor mass constraints. Progenitors appear to have a relatively small range in progenitor mass, between 8 and $17 \mathrm{M}_{\odot}$ (Smartt 2015). It is thus pertinent to ask: can such observed light-curve diversity be explained by progenitors of such similar mass?

In Fig. 2 histograms are presented of SN II absolute magnitudes and decline rates. The A14 sample is on average brighter than the direct detection sample. This is easily explained through the fact that the A14 sample is magnitude limited, while the direct 

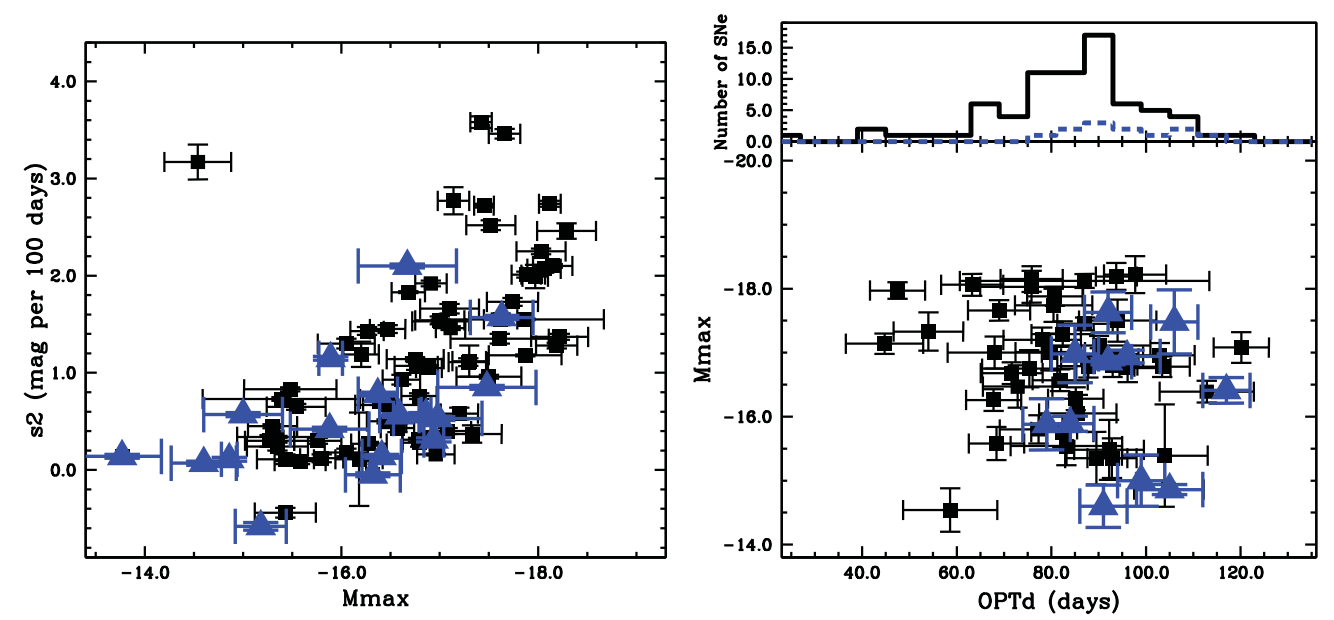

Figure 3. Left, $\boldsymbol{a})$ : SN II absolute magnitudes at maximum $\left(M_{m a x}\right)$ plotted against 'plateau' decline rate $\left(s_{2}\right)$ Right, $\left.\boldsymbol{b}\right): M_{\max }$ against optically thick phase durations (OPTds). Squares represent the distribution from A14, while triangles show SNe II with progenitor detection constraints. In the upper panel histograms for the two distributions of OPTd are also presented, solid: A14, and dashed: direct detection constraints.

detection is essentially a volume limited sample. With respect to decline rates, it is seen that there are very few direct detections that have measured $s_{1}$ declines. Fig. $2 \mathrm{~b}$ shows that there is a wide range of initial decline rates, and understanding this early behaviour could have strong constraints on SN II physics. The $s_{2}$ distribution of the direct detections is shifted towards lower values, and this is a direct consequence of the correlation between $M_{\max }$ and $s_{2}$ as presented in Fig. 3. Another interesting feature of Fig $2 \mathrm{~b}$ is the distribution of $s_{3}$ values. $s_{3}$ is the decline rate during the radioactively powered phase. Assuming full trapping of gamma-rays one expects SNe to follow a standard decline rate at these epochs. However, significant deviation from this expectation is observed. This hints at some SNe II having ejecta masses insufficient in mass and/or density to fully trap the emission.

In Fig. 3a I present $M_{\max }$ against $s_{2}$, while in Fig. 3b $M_{\max }$ against OPTd. A correlation is observed in that brighter SNe decline more quickly. A large range in OPTd values is also found, suggesting a large range in hydrogen envelope masses at the explosion epoch. Finally, I note that in all distributions -both those presented here and those in A14 and Gutiérrez et al. (2014)- an observational continuum is seen. Further statistical studies of this kind will deepen our knowledge of the SN II phenomenon and strengthen our confidence in using these explosions to probe other processed in the Universe.

\section{References}

Anderson, J. P., et al., 2014 ApJ, 786, 67

Barbon, R, Ciatti, F \& Rosino, L, 1979 A $\& A, 72,287$

Gutiérrez, C. P., et al., 2014 ApJ, 786, 15

Sanders, N. E., et al., 2015 ApJ, 799, 208

Smartt, S. J., 2015 PASA, 32, 16 